\title{
Swimming in the sea: Chemotaxis by marine bacteria
}

Justin R. Seymour*, Jean-Baptiste Raina

Climate Change Cluster (C3), University of Technology Sydney, New South Wales 2007, Australia

*email: Justin.Seymour@uts.edu.au

\begin{abstract}
Like many organisms, bacteria regularly inhabit environments characterised by spatiotemporal heterogeneity in the availability of resources required for growth and energy generation, meaning they must either tune their metabolism to prevailing conditions or have the capacity to migrate to favourable microenvironments [1]. To achieve the latter, bacteria measure their resource landscape and suitably direct their locomotion using a behaviour called chemotaxis, which is the ability to guide movement up or down chemical gradients. The capacity to perform chemotaxis is widespread across the bacterial domain, although most of our understanding of this phenotype is derived from enteric bacteria [2-3]. In the ocean, marine bacteria are often motile [4], and in fact capable of much higher swimming speeds [5] and chemotactic precision [6] than these enteric models for chemotaxis [2]. Here we discuss the underlying motives and purposes for bacterial chemotaxis in the ocean, by noting that marine bacteria experience a surprisingly heterogeneous chemical seascape [7, 8], whereby chemotaxis can provide substantial fitness advantages and even influence large-scale processes including marine ecosystem productivity, biogeochemical cycling and disease.
\end{abstract}

\section{Chemotaxis}

Motile bacteria propel themselves by rotating helical flagella driven by molecular motors $[2,3]$. Chemotaxis by these motile cells is achieved through the constant measurement of local chemical concentrations using trans-membrane chemoreceptors, while a complex signal transduction network interprets this information, allowing cells to detect chemical gradients and regulate motility accordingly [1, 2, 3]. This chemotactic behaviour ultimately allows bacteria to swim toward favourable chemicals and away from noxious substances [Figure 1]. Chemotaxis is one of the best-described sensory systems in biology, with a highly developed understanding of this behaviour acquired from well-defined model organisms, such as Escherichia coli [2]. The importance of chemotaxis is typically considered within the context of highly structured microenvironments, where chemical gradients are strong and stable, such as within biofilms or in association with the internal and external surfaces of plant and animal hosts [3]. However, growing evidence suggests chemotaxis is a ubiquitous phenotype across a wide range of environments, even within the ostensibly well-mixed and homogenous ocean, where it is employed by diverse groups of marine bacteria for a variety of functions including resource acquisition and host infection [9].

Marine bacteria use chemotaxis to exploit a heterogeneous seascape

On average, every litre of seawater contains 1 billion bacteria and several thousand 
different bacterial "species" [10]. These marine bacteria control most of the oceans" major chemical cycles (e.g. carbon, nitrogen, and sulphur) [7, 11], support food-web productivity by acting as the principal conduit for the transfer of energy and matter from the oceans' large pools of dissolved organic matter to higher trophic levels [12], and can exhibit important symbiotic and pathogenic interactions with many species of marine animals and plants $[13,14]$. However, the role of chemotaxis among marine bacteria has been widely over-looked, largely because biological oceanographers have traditionally measured microbiological processes over large spatiotemporal scales, and incorrectly presumed that the microscale chemical seascape experienced by planktonic bacteria inhabiting the ocean's water column is homogenous.

Rather than comprising a dilute homogenous soup, from the perspective of a chemotactic bacterium seawater is awash with microscale chemical features that contribute to a complex microspatial architecture [7, 15] that will often afford significant fitness advantages to chemotactic cells [26]. Large pools of suspended and sinking organic particles provide rich localized resource hotspots for bacteria that can colonise them [17], while the lysis, egestion, excretion, and exudation of other marine organisms results in a patchwork of microscale chemical gradients in the water column $[8,15]$.

Importantly, the fluid dynamics operating at these very small scales are extremely different to those experienced at larger scales, meaning that, perhaps counterintuitively, microscale chemical gradients are not significantly mixed or dispersed by ocean turbulence. Below a minimum length scale of generally a few millimetres, viscosity becomes dominant and turbulent energy is lost as heat. The only physical force acting on chemical gradients at this scale is molecular diffusion, which slowly dissipates gradients rather than mixing and erasing them [15]. It is also noteworthy that this lack of true mixing at microbial-scales means that no matter the bulk levels of ocean turbulence, the passive movement of a bacterium remains in synchrony with nearby features, so the cell will not be 'washed past' or 'washed away' from a gradient, particle or microbial associate that is suspended in the same water column [9]. Hence, the physical dynamics operating at the ocean's microscale accommodate the persistence of chemical gradients and the utility of chemotaxis in the water column.

Motile marine bacteria use chemotactic receptors to detect and target a wide range of microscale chemical features in the water column. Large pools of particulate organic carbon associated with suspended and sinking particles, ranging from micrometer-sized colloids to millimeter-sized marine snow aggregates are derived from a number of sources, including the flocculation of dead phytoplankton biomass and zooplankton fecal pellets. These particles typically contain concentrations of organic molecules that exceed background concentrations by 2 - 4 orders of magnitude [18]. Chemotactic bacteria colonize these particles at substantially higher rates than non-motile bacteria [17].

The first evidence for the potential importance of chemotaxis among marine bacteria came from the observation that many are highly chemotactic towards the chemical products of phytoplankton [19]. This led to the hypothesis that marine bacteria will use chemotaxis to colonise the "phycosphere" - the region immediately surrounding a phytoplankton cell that is enriched in exuded organic substrates [20] [Figure 2]. The potential capacity of bacteria to use chemotaxis to take advantage of this microenvironment is notable given that marine heterotrophic bacteria obtain a large 
fraction of their carbon demand directly from phytoplankton, consuming up to $50 \%$ of phytoplankton-fixed carbon [21]. Recent studies using microfluidic experiments [22] and direct microscopic observations of chemotactic bacteria aggregating around phytoplankton cells [16] support predictions that bacterial chemotaxis will enhance bacterial uptake of phytoplankton derived carbon, as well as potentially underpinning the establishment and maintenance of important phytoplankton-bacteria relationships in the ocean [20].

In addition to colonizing particles and phycospheres, there is also evidence that marine bacteria can use chemotaxis to take advantage of ephemeral microscale nutrient patches arising from the lysis and excretion of other microbes [8]. While these patches often only span a few tens to hundreds of micrometers and persist for less than 5-10 minutes, the ability of chemotactic marine bacteria to very rapidly home in on and subsequently exploit these abundant localised sources of organic substrates has been predicted to significantly enhance organic matter remineralization rates in the pelagic ocean [1].

\section{Chemotaxis at the sediment-water interface}

Compared to the complex, 3-dimensional and often short-lived chemical microenvironments occurring in the pelagic environment, the chemical landscape of the sediment-water interface at the bottom of the water column is often much simpler. Elevated concentrations of organic matter present on the seafloor promote high levels of microbial activity, which in turn generates steep vertical oxygen and nutrient gradients that are relatively stable through time. The surface sediments are often dominated by sulfur-oxidizing bacteria, including Thiovulum majus, the fastest swimming bacteria recorded (swimming up to $600 \mu \mathrm{m} \mathrm{s}^{-1}$ ) [23], which uses chemotaxis to form dense aggregations in the narrow region where optimal concentrations of oxygen and hydrogen sulphide co-exist.

\section{Host associations}

The surfaces of benthic (e.g. corals, sponges, seaweeds) and pelagic macro-organisms (e.g. fish) are also characterised by strong chemical gradients, resulting from exudation of organic and inorganic compounds, which in some cases can attract specific bacterial partners [24]. Indeed, chemotaxis is sometimes central to the establishment of host-microbe symbiotic interactions and infection by pathogens. One of the best-studied examples is the chemotaxis-mediated symbiosis between bobtail squid and the light-emitting bacterium Vibrio fischeri. Very low densities of $V$. fischeri are present in the water column, but they exhibit chemotactic migration towards chitin oligosaccharides, which are released from the squid's light-organs and act as a signal to trigger their colonisation [25]. Conversely, other vibrios are pathogens which use chemotaxis towards fish intestinal mucus ( $V$. anguillarum and $V$. furnissii) [26], or coral mucus (V. coralliilyticus and V. shiloi) [27] to initiate the infection process.

\section{From the lab to the field}

The capillary assay was the first method used to quantify chemotaxis and is still widely used today. It uses a glass capillary filled with a specific concentration of a chemoattractant, which is immersed in a homogenous bacterial suspension [28]. The compound subsequently diffuses out of the capillary and chemotactic cells respond by migrating into the capillary. A wide variety of chemotaxis assays have since been developed, but the most recent and sophisticated techniques employ microfluidic 
devices [22]. These platforms enable the generation of carefully controlled microgradients, which when coupled with high-speed video microscopy, permit tracking of the chemotactic swimming behaviour of individual cells. However, until recently, all available chemotaxis assays have shared a common limitation: their use is restricted to laboratory conditions and cultured microorgranisms. We recently developed a new device that enables assessment of chemotaxis assays among natural microbial communities of bacteria within the environment [29]. Results derived from this new in situ platform have confirmed that natural assemblages of pelagic marine bacteria exhibit strong chemotaxis towards amino-acids [29], that bacteria in the vicinity of reef-building corals are attracted by common coral exudates such as dimethylsulfoniopropionate [30], and that the acquisition of specific bacterial associates by marine sponges is not solely passive, but can also be mediated by chemotaxis [31].

\section{The importance of chemotaxis in the marine environment}

Besides providing a competitive advantage to motile cells, chemotaxis among marine bacteria has the potential to impact a number of large-scale ecological and biogeochemical processes. The initiation of symbiotic interactions that underpin the ecological succuss of some of the most productive marine ecosystems (e.g. coral reefs, hydrothermal vents, seagrass meadows) often relies on chemotaxis [13, 30]. This behaviour is also involved in pathogenicity and disease outbreaks among a wide range of marine species [33], both in natural habitats [27] and in aquaculture settings [34], sometimes with dire ecological and economic consequences. In the pelagic environment, chemotaxis plays an often pivotal role in all major biogeochemical cycles, by affecting the rates and directions of key chemical transformations $[1,9$, 20]. For example, chemotaxis-mediated particle colonisation influences controls the amount of carbon that either sinks to the deep sea or is respired in the upper ocean, ultimately influencing global carbon budgets [35].

\section{Summary}

Rather than the homogenous microscale seascape long assumed by oceanographers, marine bacteria inhabit a surprisingly heterogeneous environment, with a plethora of localized microniches likely to persist in the water column, across spatial scales commensurate with the movement of motile bacteria. The capacity to employ chemotaxis to navigate this environment and successfully exploit microscale resource gradients will therefore provide a significant fitness advantage to some marine bacteria within a number scenarios. Many examples of highly effective chemotactic capacity among both cultured marine bacteria and natural communities are consistent with this notion, with several lines of evidence suggesting that these microscale bacterial behaviour's will likely have a number of important ocean-scale implications.

\section{Figures}

Figure 1: During chemotaxis, motile bacteria sense chemical concentrations using transmembrane chemoreceptors allowing them to direct their movement up or down chemical gradients, often resulting in accumulation of cells near to the source of a chemical attractant.

Figure 2: The phycosphere is the region surrounding an individual phytoplankton cell that is enriched in dissolved organic substrates exuded by the cell into the surrounding 
water. Chemical gradients within the phycosphere are utilized by chemotactic bacteria to colonise this microenvironment in order to gain increased access to phytoplanktonderived organic carbon and/or establish more specific metabolic interactions with the phytoplankton cell.

Acknowledgements: This work was supported by an Australian Research Council (ARC) Discovery Project DP140101045 and Gordon and Betty Moore Foundation Grant GBMF3801 to J.R.S. J.R.S is supported by ARC Future Fellowship FT130100218 and J-B.R is supported by ARC DECRA Fellowship DE160100636.

\section{Author Biographies:}

Justin Seymour is an ARC Future Fellow and Associate Professor at the University of Technology Sydney, where he leads the Ocean Microbiology Group. His research is focused on the ecology of marine microorganisms and among his interests is how marine bacteria use behaviours typically considered most important in highly structured and confined environments - namely motility and chemotaxis - to exploit a patchy resource landscape within the ocean's water column.

Jean-Baptiste Raina is an ARC DECRA Fellow at the University of Technology of Sydney. His research focuses on marine microbial symbioses and in his current project he is developing new tools to visualize and quantify the interactions occurring between marine microbes at the micrometer-scale.

\section{References:}

1. Fenchel, T. (2002) Microbial behavior in a heterogeneous world. Science 296 (5570), 1068-1071.

2. Berg, H. (2004) E. coli in motion, Springer.

3. Wadhams, G.H. and Armitage, J.P. (2004) Making sense of it all: bacterial chemotaxis. Nature Reviews Molecular Cell Biology 5, 1024.

4. Grossart, H.P. et al. (2001) Bacterial motility in the sea and its ecological implications. Aquatic Microbial Ecology 25 (3), 247-258.

5. Mitchell, J.G. et al. (1995) Natural assemblages of marine bacteria exhibiting highspeed motility and large accelerations. Applied and Environmental Microbiology 61 (12), 4436-40.

6. Son, K. et al. (2016) Speed-dependent chemotactic precision in marine bacteria. Proceedings of the National Academy of Sciences 113 (31), 8624-8629.

7. Azam, F. (1998) Microbial control of oceanic carbon flux: the plot thickens. Science 280 (5364), 694-696.

8. Blackburn, N. et al. (1998) Microscale nutrient patches in planktonic habitats shown by chemotactic bacteria. Science 282 (5397), 2254-2256.

9. Stocker, R. and Seymour, J.R. (2012) Ecology and physics of bacterial chemotaxis in the ocean. Microbiology and Molecular Biology Reviews 76 (4), 792-812.

10. Sogin, M.L. et al. (2006) Microbial diversity in the deep sea and the underexplored "rare biosphere". Proceedings of the National Academy of Sciences 103 (32), 12115-12120.

11. Falkowski, P.G. et al. (2008) The microbial engines that drive Earth's biogeochemical cycles. Science 320 (5879), 1034-1039. 
12. Azam, F. et al. (1983) The ecological role of water column microbes in the sea. Marine Ecology Progress Series 10, 257-263.

13. Rosenberg, E. et al. (2007) The role of microorganisms in coral health, disease and evolution. Nature Reviews Microbiology 5, 355.

14. Webster, N. et al. (2013) Same, same but different: symbiotic bacterial associations in GBR sponges. Frontiers in Microbiology 3 (444).

15. Stocker, R. (2012) Marine microbes see a sea of gradients. Science 338 (6107), 628-633.

16. Smriga, S. et al. (2016) Chemotaxis toward phytoplankton drives organic matter partitioning among marine bacteria. Proceedings of the National Academy of Sciences 113 (6), 1576-1581.

17. Kiørboe, T. et al. (2002) Mechanisms and rates of bacterial colonization of sinking aggregates. Applied and Environmental Microbiology 68 (8), 3996-4006. 18. Prgzelin, B.B. and Alldredge, A.L. (1983) Primary production of marine snow during and after an upwelling event1. Limnology and Oceanography 28 (6), 11561167.

19. Bell, W. and Mitchell, R. (1972) Chemotactic and growth responses of marine bacteria to algal extracellular products. Biological Bulletin 143 (2), 265-277.

20. Seymour, J.R. et al. (2017) Zooming in on the phycosphere: the ecological interface for phytoplankton-bacteria relationships. Nature Microbiology 2, 17065. 21. Fuhrman, J.A. and Azam, F. (1982) Thymidine incorporation as a measure of heterotrophic bacterioplankton production in marine surface waters: Evaluation and field results. Marine Biology 66 (2), 109-120.

22. Seymour, J. et al. (2008) A microfluidic chemotactic assay for assessing the behavior of microbes within diffusing nutrient patches. Limnology and Oceanography: Methods 6, 477-488.

23. Jørgensen, B.B. and Revsbech, N.P. (1983) Colorless sulfur bacteria, Beggiatoa spp. and Thiovulum spp., in $\mathrm{O}_{2}$ and $\mathrm{H}_{2} \mathrm{~S}$ microgradients. Applied and Environmental Microbiology 45 (4), 1261-1270.

24. Ottemann, K.M. and Miller, J.F. (1997) Roles for motility in bacterial-host interactions. Molecular Microbiology 24 (6), 1109-1117.

25. DeLoney-Marino, C.R. et al. (2003) Chemoattraction of Vibrio fischeri to serine, nucleosides, and $\mathrm{N}$-acetylneuraminic acid, a component of squid light-organ mucus. Applied and Environmental Microbiology 69 (12), 7527-7530.

26. O'Toole, R. et al. (1996) Chemotactic motility is required for invasion of the host by the fish pathogen Vibrio anguillarum. Molecular Microbiology 19 (3), 625-637.

27. Garren, M. et al. (2014) A bacterial pathogen uses dimethylsulfoniopropionate as a cue to target heat-stressed corals. ISME J 8 (5), 999-1007.

28. Adler, J. and Dahl, M.M. (1967) A method for measuring the motility of bacteria and for comparing random and non-random motility. Microbiology 46 (2), 161-173. 29. Lambert, B.S. et al. (2017) A microfluidics-based in situ chemotaxis assay to study the behaviour of aquatic microbial communities. Nature Microbiology 2 (10), 1344-1349.

30. Tout, J. et al. (2015) Chemotaxis by natural populations of coral reef bacteria. The Isme Journal 9, 1764.

31. Tout, J. et al. (2017) Redefining the sponge-symbiont acquisition paradigm:

sponge microbes exhibit chemotaxis towards host-derived compounds. Environmental Microbiology Reports 9 (6), 750-755.

33. Butler, S.M. and Camilli, A. (2005) Going against the grain: chemotaxis and infection in Vibrio cholerae. Nature Reviews Microbiology 3, 611. 
34. Declercq, A.M. et al. (2013) Columnaris disease in fish: a review with emphasis on bacterium-host interactions. Veterinary Research 44 (1), 27.

35. Azam, F. and Long, R.A. (2001) Sea snow microcosms. Nature 414, 495. 